\title{
A Case of COVID-19 Complicated by Venous Thromboembolism Despite Being on Prophylactic Anticoagulation
}

\author{
Amit Rout ${ }^{\mathrm{a}, \mathrm{b}}$, Abigail Chan ${ }^{\mathrm{a}}$
}

\begin{abstract}
The coronavirus disease 2019 (COVID-19) presents with variable clinical syndromes, from asymptomatic disease to acute respiratory failure. Complications such as acute respiratory distress syndrome (ARDS), secondary infection, acute cardiac injury, liver failure, renal failure, coagulable disorders and in many cases, death have been recognized. Venous thromboembolism (VTE) was found to be present in up to one-third of critically ill COVID-19 patients. Our case demonstrated a patient with COVID-19 who developed a massive pulmonary embolism leading to cardiac arrest, despite having an initial normal D-dimer level, normal chest imaging, and while maintained on appropriate VTE prophylaxis during hospitalization.
\end{abstract}

Keywords: COVID-19; SARS-Cov-2; Venous thromboembolism

\section{Introduction}

The world is currently going through one of the biggest medical challenges of the 21st century, the coronavirus disease 2019 (COVID-19). With the advent of COVID-19, variable clinical presentations from asymptomatic to severe respiratory symptoms requiring intensive care unit (ICU) admissions have been seen. Complications such as acute respiratory distress syndrome (ARDS), secondary infection, acute cardiac injury, liver failure, renal failure, coagulable disorders and in many cases, death have been recognized [1]. Venous thromboembolism (VTE) has been reported in $25-31 \%$ of critically ill patients $[2,3]$. We present a case of 62 -year-old man diagnosed with COVID-19 pneumonia and respiratory failure whose course was complicated by cardiac arrest secondary to VTE.

Manuscript submitted May 18, 2020, accepted May 26, 2020

Published online June 18, 2020

aDepartment of Internal Medicine, Sinai Hospital of Baltimore, Baltimore, MD 21215, USA

${ }^{b}$ Corresponding Author: Amit Rout, Department of Internal Medicine, Sinai Hospital of Baltimore, $2401 \mathrm{~W}$ Belvedere Avenue, Baltimore, MD 21215, USA. Email: amitrout90@gmail.com

doi: https://doi.org/10.14740/jmc3500

\section{Case Report}

A 62-year-old man with underlying obstructive sleep apnea, hyperlipidemia, and depression, presented to the emergency department with shortness of breath, cough, and fever for the last 3 days. His wife was admitted at another institution with COVID-19 pneumonia. On arrival, he was afebrile, normal heart rate and blood pressure, slightly tachypneic, with oxygen saturation of $88 \%$ on room air. He was awake and conversant. Physical examination revealed mild respiratory distress, diffusely coarse breath sounds, no jugular vein distention, distinct heart sounds, and no peripheral edema.

Pertinent laboratory findings were elevated: lactate dehydrogenase (LDH) of $422 \mathrm{U} / \mathrm{L}$ (normal value 84 - $246 \mathrm{U} / \mathrm{L}$ ), ferritin of $449 \mathrm{ng} / \mathrm{mL}$ (normal values $28-365 \mathrm{ng} / \mathrm{mL}$ ), C-reactive protein of $106 \mathrm{mg} / \mathrm{L}$ (normal values $\leq 9.9 \mathrm{mg} / \mathrm{L}$ ), procalcitonin $0.14 \mathrm{ng} /$ $\mathrm{mL}$ (normal values $\leq 0.08 \mathrm{ng} / \mathrm{mL}$ ). His troponin, complete blood count $(\mathrm{CBC})$, creatinine, and liver function tests were normal. His prothrombin time (PT) and international normalized ratio (INR) were normal. His activated partial thromboplastin time (aPTT) was mildly prolonged $35.3 \mathrm{~s}$ (normal values $23-26.1 \mathrm{~s}$ ). D-dimer was $0.48 \mathrm{mg} / \mathrm{dL}$ (normal values $\leq 0.5 \mathrm{mg} / \mathrm{dL}$ ). His COVID-19 polymerase chain reaction (PCR) testing was positive. Chest Xray showed multifocal interstitial and airspace opacities bilaterally without any pleural effusion which was confirmed with computed tomography (CT) angiogram of the thorax (Fig. 1). There was no evidence of pulmonary embolism. Electrocardiogram (ECG) on admission was normal sinus rhythm with $\mathrm{T}$ wave inversion in leads V1, V2 and V3, which was similar to a previous ECG.

Initially, he was placed on nasal cannula at $6 \mathrm{~L} / \mathrm{min}$ which was quickly advanced to mechanical ventilation given worsening hypoxemia. He was treated with empiric antibiotics and hydroxychloroquine. VTE prophylaxis was initiated using subcutaneous heparin and mechanical compression devices. During his hospital stay he was managed with mechanical proning and paralytics as per institutional COVID-19 ARDS protocol. He was slowly showing improvement while remaining on mechanical ventilator support by the day 10 of hospitalization. On the 11th hospital day, he became tachycardic, with heart rate up to the 130's. ECG showed a new right bundle branch block with S1Q3T3 pattern (Fig. 2) and elevated troponin. Prior to undergoing planned CT angiogram of thorax and Doppler ultrasonography to rule out VTE, he went into cardiac arrest. Advanced cardiac life support was started. The point-of-care ultrasonography 


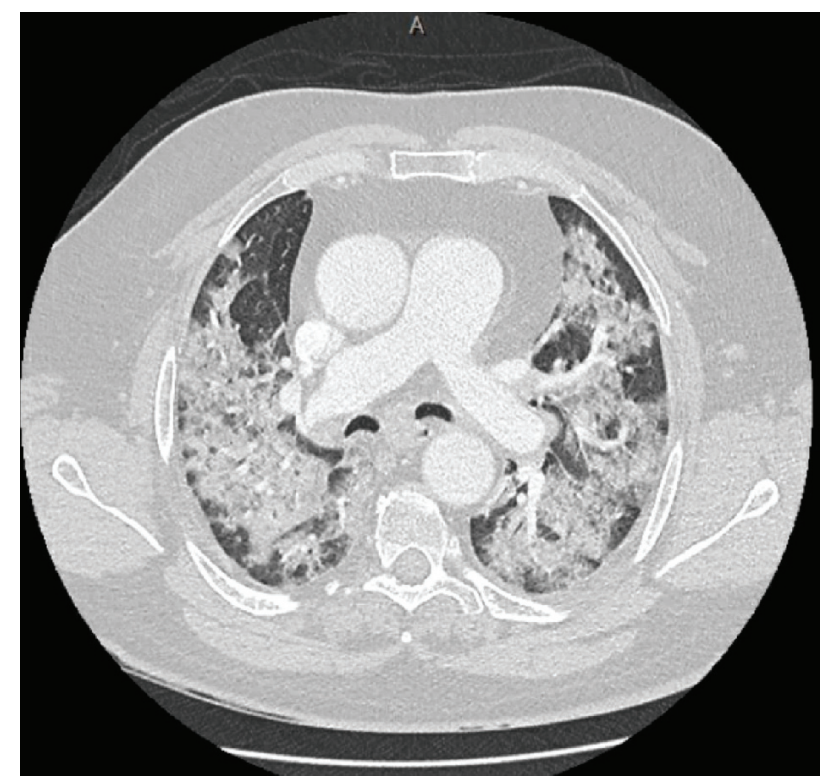

Figure 1. Computed Tomography angiogram of the thorax showing extensive bilateral patchy and ground glass opacities throughout the lungs without any pulmonary embolism.

of the heart revealed dilated right ventricle, thus systemic tissue plasminogen activator (alteplase) was administered for probable massive pulmonary embolism. Patient had a transient return of circulation and was started on intravenous (IV) vasopressor support. A bedside Doppler ultrasonography demonstrated deep vein thrombosis in bilateral lower extremities thus confirming diagnosis of VTE (Fig. 3). Intravenous heparin infusion was started. Despite maximum support, patient expired.

\section{Discussion}

This case demonstrates patients with COVID-19 are prone to develop large VTE which can lead to cardiac arrest, which is despite having an initial normal D-dimer level, imaging and while being on appropriate VTE prophylaxis during hospitalization.

Approximately one-third of COVID-19 patients are being diagnosed with VTE. Autopsy findings of some COVID-19 patients revealed both micro- and macro-thrombi formation [4]. Pharmacologic VTE prophylaxis is recommended unless with high bleeding risks [2-6]. Patients with high risk for VTE are those who are critically ill, undergoing cytokine storm syndrome, having high levels of D-dimer, abnormal coagulation markers, and experiencing multi-organ failure. The use of empiric therapeutic doses of anticoagulation, higher doses of pharmacologic VTE prophylaxis, and post-discharge thromboprophylaxis in high-risk patients are still being investigated [4, 7]. Currently, a high level of D-dimer on admission is being used to determine patients with an increased risk for VTE. A study by Cui et al proposed a D-dimer threshold of $>1.5 \mathrm{mg} / \mathrm{dL}$ to determine VTE risk, with sensitivity and specificity rates of $85 \%$ and $89 \%$, respectively. They found that patients who present with VTE tend to be older, have prolonged aPTT, lymphopenia, and higher Ddimer levels (5.2 vs. $1.2 \mathrm{mg} / \mathrm{mL}, \mathrm{P}<0.001)$ when compared to patients without VTE [2]. Serial laboratory markers especially D-dimer and imaging for VTE should be considered in patients with severe COVID-19 disease. Use of prophylactic anticoagulation versus therapeutic anticoagulation in these patients with negative imaging for VTE remains controversial. Full dose anticoagulation is associated with increased risk of bleeding, thus before recommending empiric use further studies are needed to establish mortality benefits.

\section{Conclusions}

Patients with COVID-19 who are on the severe side of the disease spectrum have increased risk of VTE despite being on prophylactic dose of anticoagulation. A high index of suspicion for VTE should be maintained all the time. Further studies evaluating empiric initiation of therapeutic anticoagulation is needed.

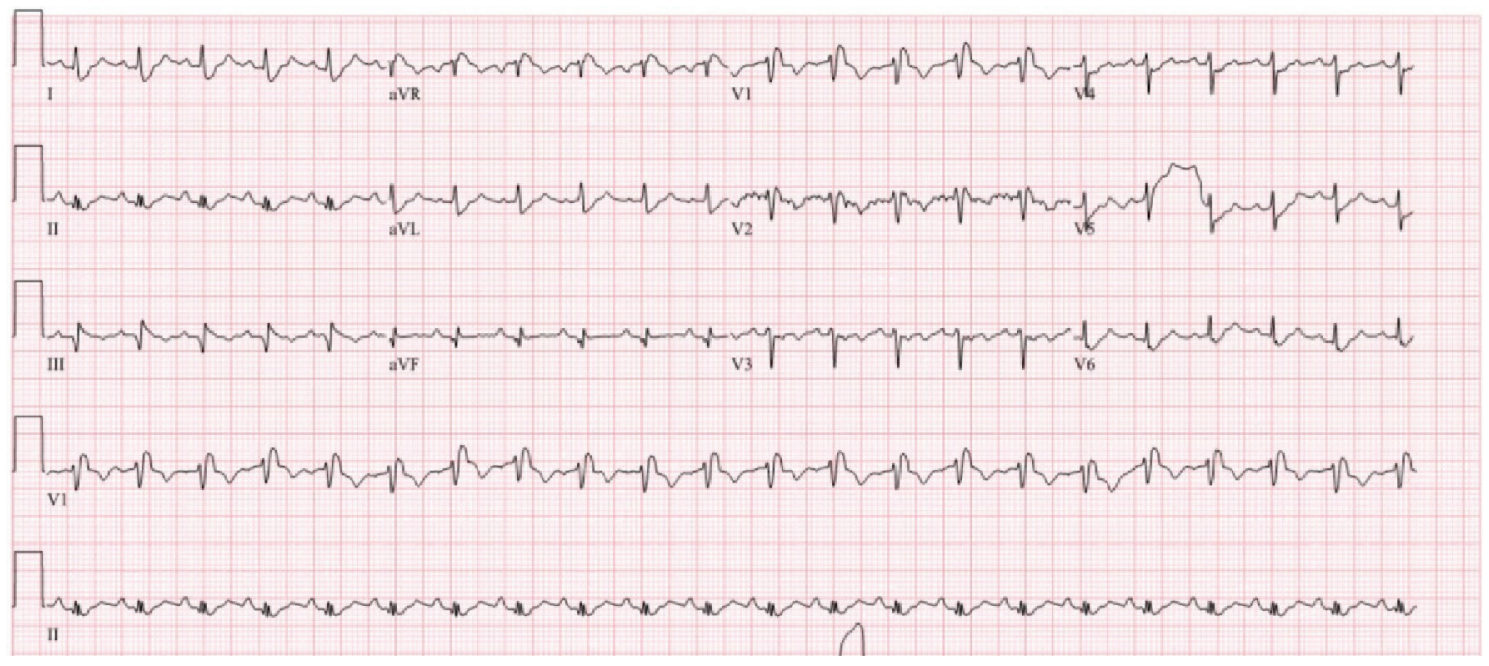

Figure 2. Electrocardiogram, obtained before cardiac arrest showing sinus tachycardia, S1Q3T3 pattern and a new right bundle branch block. 

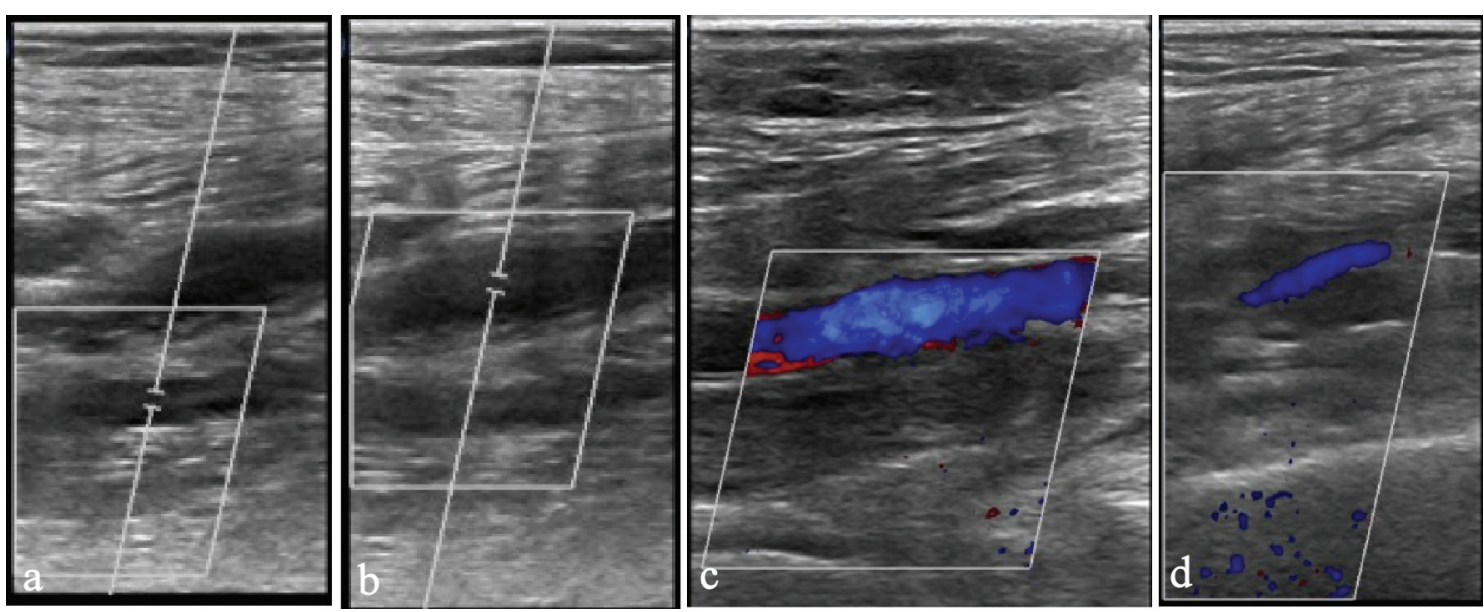

Figure 3. Doppler ultrasonography of lower extremity. Non-compressible and diminished Doppler signal of right peroneal (a), right posterior tibial veins (b), left peroneal (c), and left posterior tibial veins (d) indicating thrombi.

\section{Acknowledgments}

None to declare.

\section{Financial Disclosure}

None to declare.

\section{Conflict of Interest}

None to declare.

\section{Informed Consent}

Not applicable.

\section{Author Contributions}

$\mathrm{AR}$ and $\mathrm{AC}$ contributed equally in conceptualizing, data reviewing and writing of this manuscript.

\section{Data Availability}

The authors declare that data supporting the findings of this study are available within the article.

\section{References}

1. Chen G, Wu D, Guo W, Cao Y, Huang D, Wang H, Wang $\mathrm{T}$, et al. Clinical and immunological features of severe and moderate coronavirus disease 2019. J Clin Invest. 2020;130(5):2620-2629.

2. Cui S, Chen S, Li X, Liu S, Wang F. Prevalence of venous thromboembolism in patients with severe novel coronavirus pneumonia. J Thromb Haemost. 2020.

3. Klok FA, Kruip M, van der Meer NJM, Arbous MS, Gommers D, Kant KM, Kaptein FHJ, et al. Incidence of thrombotic complications in critically ill ICU patients with COVID-19. Thromb Res. 2020.

4. Castelli R, Gidaro A. Abnormal Hemostatic Parameters and Risk of Thromboembolism Among Patients With COVID-19 Infection. J Hematol. 2020;9(1-2):1-4.

5. Thachil J, Tang N, Gando S, Falanga A, Cattaneo M, Levi M, Clark C, et al. ISTH interim guidance on recognition and management of coagulopathy in COVID-19. J Thromb Haemost. 2020;18(5):1023-1026.

6. Tang N, Li D, Wang X, Sun Z. Abnormal coagulation parameters are associated with poor prognosis in patients with novel coronavirus pneumonia. J Thromb Haemost. 2020;18(4):844-847.

7. Kreuziger L, Lee A, Garcia D, et al. Accessed on April 17, 2020. COVID-19 and VTE/Anticoagulation: frequently asked questions. Retrieved from https://www.hematology.org/covid-19/covid-19-and-vte-anticoagulation. 